CRYSTALLOGRAPHIC COMMUNICATIONS
Received 30 March 2021

Accepted 6 April 2021

Edited by L. Van Meervelt, Katholieke Universiteit Leuven, Belgium

Keywords: crystal structure; tetrahydroisoquinoline; hydrogen bond; twist-boat conformation; Hirshfeld surface analysis.

CCDC reference: 2075592

Supporting information: this article has supporting information at journals.iucr.org/e

\section{Crystal structure and Hirshfeld surface analysis of 2-\{[7-acetyl-8-(4-chlorophenyl)-4-cyano-6-hydroxy- 1,6-dimethyl-5,6,7,8-tetrahydroisoquinolin-3- yl]sulfanyl\}-N-(4-chlorophenyl)acetamide}

\author{
Mehmet Akkurt, ${ }^{\text {a Islam S. Marae, }}{ }^{\text {b Joel T. Mague, }}{ }^{\text {c }}$ Shaaban K. Mohamed, ${ }^{\text {de }}$ Etify A. \\ Bakhite $^{b}$ and Safiyyah A. H. Al-Waleedy ${ }^{f_{*}}$
}

${ }^{\mathbf{a}}$ Department of Physics, Faculty of Sciences, Erciyes University, 38039 Kayseri, Turkey, ${ }^{\mathbf{b}}$ Chemistry Department, Faculty

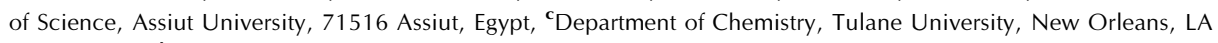
70118, USA, ${ }^{\mathbf{d}}$ Chemistry and Environmental Division, Manchester Metropolitan University, Manchester, M1 5GD, England, ${ }^{\mathbf{e}}$ Chemistry Department, Faculty of Science, Minia University, 61519 El-Minia, Egypt, and ${ }^{\mathbf{f} D e p a r t m e n t ~ o f ~}$ Chemistry, Faculty of Science, Taiz University, Taiz, Yemen. *Correspondence e-mail: shaabankamel@yahoo.com

In the title molecule, $\mathrm{C}_{28} \mathrm{H}_{25} \mathrm{Cl}_{2} \mathrm{~N}_{3} \mathrm{O}_{3} \mathrm{~S}$, the heterocyclic portion of the tetrahydroisoquinoline unit is planar while the cyclohexene ring adopts a twist-boat conformation. The two 4-chlorophenyl groups extend away from one side of this unit while the hydroxyl and acetyl groups extend away from the opposite side and form an intramolecular $\mathrm{O}-\mathrm{H} \cdots \mathrm{O}$ hydrogen bond. The crystal packing consists of layers parallel to the $b c$ plane. A Hirshfeld surface analysis of the crystal structure indicates that the most important contributions to the crystal packing are from $\mathrm{H} \cdots \mathrm{H}(37.3 \%), \mathrm{Cl} \cdots \mathrm{H} / \mathrm{H} \cdots \mathrm{Cl}(17.6 \%), \mathrm{O} \cdots \mathrm{H} /$ $\mathrm{H} \cdots \mathrm{O}(11.1 \%), \mathrm{C} \cdots \mathrm{H} / \mathrm{H} \cdots \mathrm{C}(10.9 \%)$ and $\mathrm{N} \cdots \mathrm{H} / \mathrm{H} \cdots \mathrm{N}(9.7 \%)$ interactions.

\section{Chemical context}

The tetrahydroisoquinoline motif is present in a variety of natural products, including cactus alkaloids (peyoruvic acid; Chrzanowska et al., 1987) and mammalian alkaloids (salsoline carboxylic acid; Czarnocki et al., 1992). Biological tests indicate that tetrahydroisoquinolines can act as bronchodilators (Houston \& Rodger, 1974) and anticonvulsants (Ohkubo et al., 1996; Thompson et al., 1990) and they have also shown anti-hypoxic activity (Gill et al., 1991). Based on these findings and following our interest in this area, we herein report the synthesis and crystal structure of the title compound.

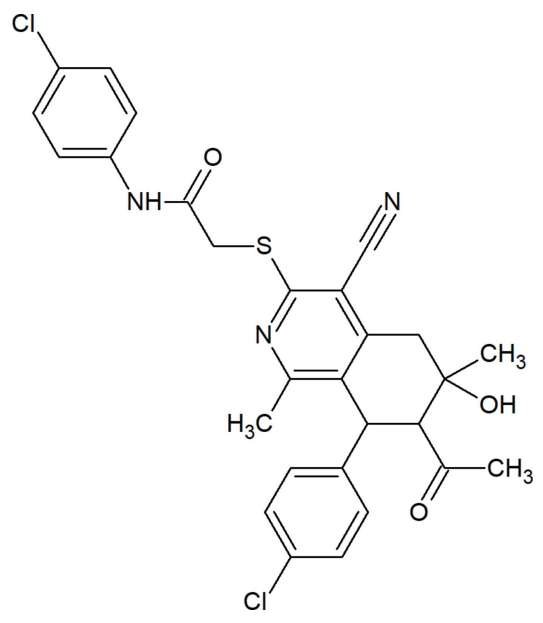




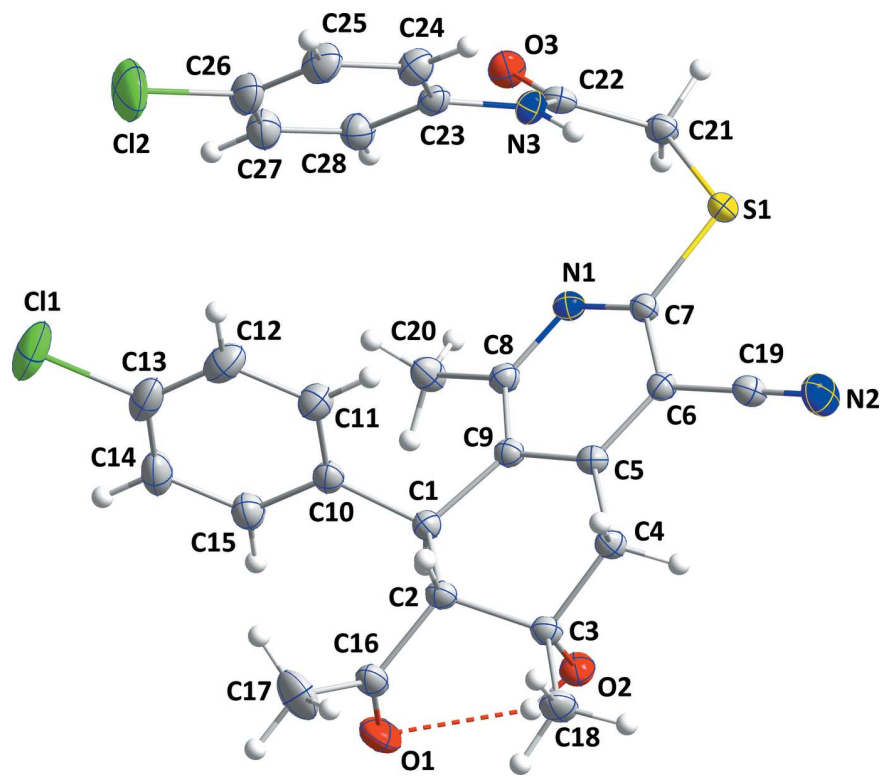

Figure 1

The title molecule with the labeling scheme and $50 \%$ probability ellipsoids. The intramolecular $\mathrm{O}-\mathrm{H} \cdots \mathrm{O}$ hydrogen bond is depicted by a dashed line.

\section{Structural commentary}

The overall conformation of the title molecule, Fig. 1, resembles that of a chair with the tetrahydroisoquinoline core forming the seat, the hydroxyl and acetyl oxygen atoms forming stubby legs and the 4-chlorophenyl group and the amide group forming the back. The N1/C5-C9 ring is essentially planar (r.m.s. deviation $=0.041 \AA$ ) with the largest deviation of 0.059 (1) $\AA$ being for atom C9. A puckering analysis (Cremer \& Pople, 1975) of the $\mathrm{C} 1-\mathrm{C} 5 / \mathrm{C} 9$ ring yielded the following parameters: $Q_{\mathrm{T}}=0.5230(13) \AA, \theta=54.39(14)^{\circ}$ and $\varphi=96.94(17)^{\circ}$. The conformation of this ring approximates a twist-boat conformation. The best planes through the $\mathrm{C} 10-\mathrm{C} 15$ and $\mathrm{C} 23-\mathrm{C} 28$ rings are inclined to the $\mathrm{N} 1 / \mathrm{C} 5-\mathrm{C} 9$ plane by $76.05(6)$ and $74.04(6)^{\circ}$, respectively. The acetyl

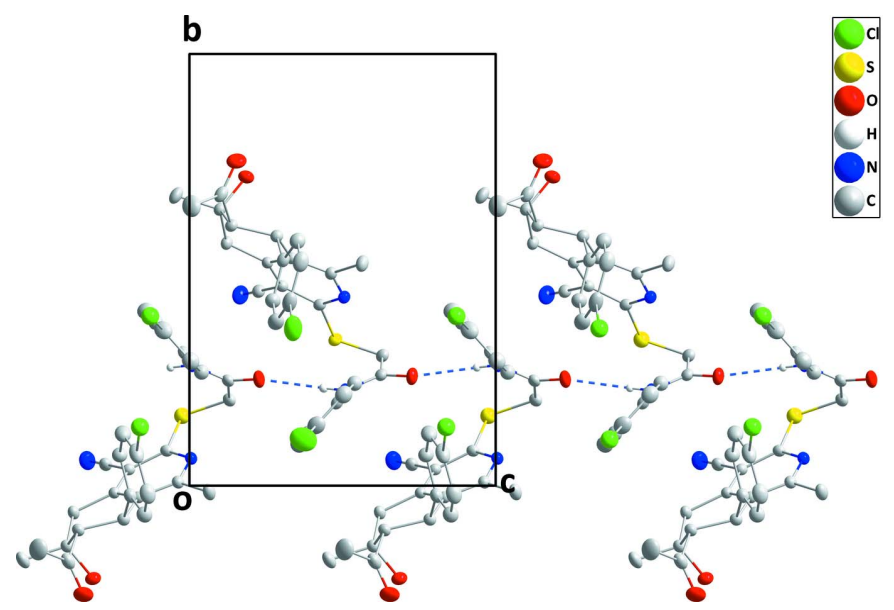

Figure 2

A portion of one chain viewed along the $a$-axis direction with the intermolecular $\mathrm{N}-\mathrm{H} \cdots \mathrm{O}$ hydrogen bonds depicted by dashed lines.
Table 1

Hydrogen-bond geometry $\left(\AA,{ }^{\circ}\right)$.

\begin{tabular}{lllll}
\hline$D-\mathrm{H} \cdots A$ & $D-\mathrm{H}$ & $\mathrm{H} \cdots A$ & $D \cdots A$ & $D-\mathrm{H} \cdots A$ \\
\hline $\mathrm{O} 2-\mathrm{H} 2 A \cdots \mathrm{O} 1$ & 0.87 & 2.14 & $2.8746(14)$ & 142 \\
$\mathrm{~N} 3-\mathrm{H} 3 \cdots \mathrm{O} 3^{\mathrm{i}}$ & 0.91 & 2.17 & $2.9362(13)$ & 141 \\
\hline
\end{tabular}

Symmetry code: (i) $x,-y+\frac{1}{2}, z-\frac{1}{2}$.

group on $\mathrm{C} 2$ is in an equatorial position while the hydroxyl group on $\mathrm{C} 3$ is axial and these are syn to one another. The C10-C15 ring attached to $\mathrm{C} 1$ is close to equatorial and anti with respect to both other substituents (Table 1, Fig. 1). The $\mathrm{O} 2-\mathrm{H} 2 A$ hydroxyl group is favorably oriented for forming an intramolecular hydrogen bond with O1 (Fig. 1). This was not seen for some related molecules where a stronger intermolecular interaction is favored for these $\mathrm{O}$ atoms (Al-Taifi et al., 2021).

\section{Supramolecular features and Hirshfeld surface analysis}

In the crystal, helical chains extending along the $c$-axis direction are formed by $\mathrm{N} 3-\mathrm{H} 3 \cdots \mathrm{O} 3$ hydrogen bonds (Table 1 and Fig. 2). Inversion-related chains pack together to form thick layers, which have the chlorine atoms on the outsides (Fig. 3). In addition, a $\mathrm{C} 22-\mathrm{O} 3 \cdots \mathrm{Cg} 1^{\mathrm{ii}}$ interaction $\left[\mathrm{C} 22-\mathrm{O} 3=1.3576(15) \AA\right.$, O3 $\cdots C g 1^{\mathrm{ii}}=3.6287$ (11) $\AA$ and $\mathrm{C} 22-\mathrm{O} 3 \cdots \mathrm{Cg} 1^{\mathrm{ii}}=115.38(8)^{\circ}$; symmetry code: (ii) $x, \frac{1}{2}-y$, $\frac{1}{2}+z$; where $C g 1$ is the centroid of the N1/C5-C9 ring) are also observed in the crystal structure.

The intermolecular interactions in the crystal of the title compound were investigated and visualized by performing a Hirshfeld surface analysis (Spackman \& Jayatilaka, 2009) using Crystal Explorer 17.5 (Turner et al., 2017). The Hirshfeld surface plotted over $d_{\text {norm }}$ in the range -0.3918 to +1.6138 a.u. is shown in Fig. 4 with red areas indicating distances shorter (in closer contact) and blue those longer (distant contact) than the van der Waals separations. The closest contacts are listed

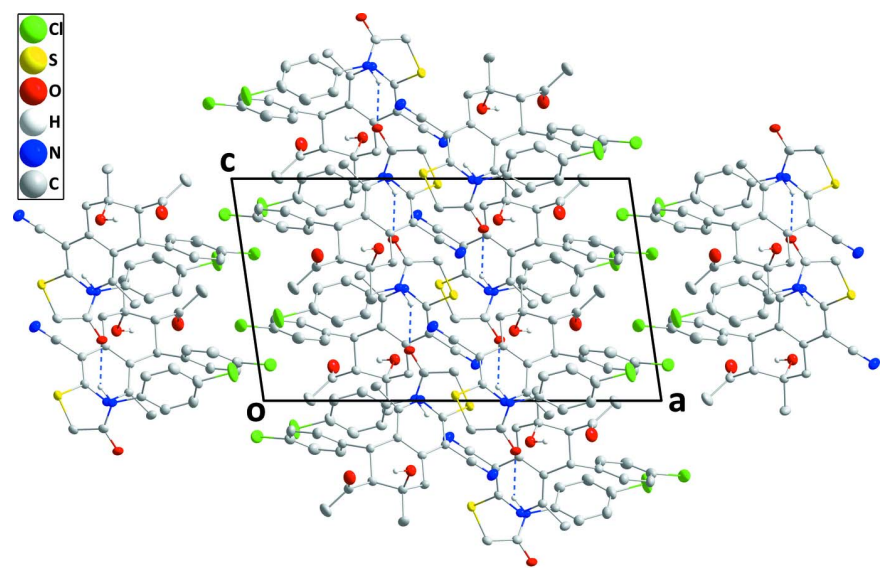

Figure 3

Packing viewed along the $b$-axis direction with $\mathrm{N}-\mathrm{H} \cdots \mathrm{O}$ hydrogen bonds depicted by dashed lines. 
Table 2

Summary of short interatomic contacts $(\AA)$ in the title compound.

\begin{tabular}{lll}
\hline Contact & Distance & Symmetry operation \\
\hline $\mathrm{C} 12 \cdots \mathrm{H} 14$ & 3.06 & $-x,-\frac{1}{2}+y, \frac{1}{2}-z ;$ \\
$\mathrm{H} 14 \cdots \mathrm{Cl} 1$ & 2.98 & $-x, 1-y, 1-z ;$ \\
$\mathrm{H} 17 A \cdots \mathrm{Cl} 1$ & 3.02 & $-x, 1-y,-z ;$ \\
$\mathrm{S} 1 \cdots \mathrm{H} 18 B$ & 3.17 & $1-x,-\frac{1}{2}+y, \frac{1}{2}-z ;$ \\
$\mathrm{H} 21 B \cdots \mathrm{H} 4 A B$ & 2.51 & $1-x, 1-y, 1-z ;$ \\
$\mathrm{H} 3 \cdots \mathrm{O} 3$ & 2.17 & $x, \frac{1}{2}-y,-\frac{1}{2}+z ;$ \\
$\mathrm{H} 1 \cdots \mathrm{H} 18 C$ & 2.26 & $x, \frac{3}{2}-y, \frac{1}{2}+z ;$ \\
$\mathrm{H} 18 B \cdots \mathrm{N} 2$ & 2.86 & $1-x, 1-y,-z$. \\
\hline
\end{tabular}

Table 3

Percentage contributions of interatomic contacts to the Hirshfeld surface for the title compound.

\begin{tabular}{ll}
\hline Contact & Percentage contribution \\
\hline $\mathrm{H} \cdots \mathrm{H}$ & 37.3 \\
$\mathrm{Cl} \cdots \mathrm{H} / \mathrm{H} \cdots \mathrm{Cl}$ & 17.6 \\
$\mathrm{O} \cdots \mathrm{H} / \mathrm{H} \cdots \mathrm{O}$ & 11.1 \\
$\mathrm{C} \cdots \mathrm{H} / \mathrm{H} \cdots \mathrm{C}$ & 10.9 \\
$\mathrm{~N} \cdots \mathrm{H} / \mathrm{H} \cdots \mathrm{N}$ & 9.7 \\
$\mathrm{~S} \cdots \mathrm{H} / \mathrm{H} \cdots \mathrm{S}$ & 2.9 \\
$\mathrm{C} \cdots \mathrm{C} / \mathrm{C} \cdots \mathrm{Cl}$ & 1.7 \\
$\mathrm{O} \cdots \mathrm{C} / \mathrm{C} \cdots \mathrm{O}$ & 1.6 \\
$\mathrm{~S} \cdots \mathrm{C} / \mathrm{C} \cdots \mathrm{S}$ & 1.6 \\
$\mathrm{Cl} \cdots \mathrm{O} / \mathrm{O} \cdots \mathrm{Cl}$ & 1.6 \\
$\mathrm{C} \cdots \mathrm{C}$ & 1.3 \\
$\mathrm{~N} \cdots \mathrm{C} / \mathrm{C} \cdots \mathrm{N}$ & 1.1 \\
$\mathrm{~S} \cdots \mathrm{O} / \mathrm{O} \cdots \mathrm{S}$ & 0.8 \\
$\mathrm{~S} \cdots \mathrm{N} / \mathrm{N} \cdots \mathrm{S}$ & 0.4 \\
$\mathrm{~N} \cdots \mathrm{N}$ & 0.2 \\
$\mathrm{Cl} \cdots \mathrm{Cl}$ & 0.2 \\
$\mathrm{O} \cdots \mathrm{N} / \mathrm{N} \cdots \mathrm{O}$ & 0.1 \\
\hline
\end{tabular}

in Table 2. The $\mathrm{O}-\mathrm{H} \cdots \mathrm{O}$ and $\mathrm{N}-\mathrm{H} \cdots \mathrm{O}$ hydrogen bonds are clearly shown by the dark-red circles (Tables 1 and 2; Fig. 4).

Fig. 5 shows the full two-dimensional fingerprint plot (McKinnon et al., 2007) and those delineated into the major contacts: $\mathrm{H} \cdots \mathrm{H}$ (37.3\%; Fig. $5 b), \mathrm{Cl} \cdots \mathrm{H} / \mathrm{H} \cdots \mathrm{Cl}(17.6 \%$;

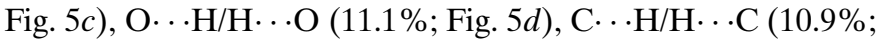
Fig. $5 e)$ and $\mathrm{N} \cdots \mathrm{H} / \mathrm{H} \cdots \mathrm{N}(9.7 \%$; Fig. $5 f)$. The other contacts are negligible with individual contributions of less than $2.9 \%$ and are given in Table 3.

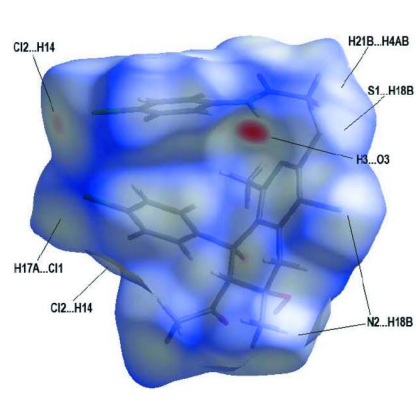

(a)

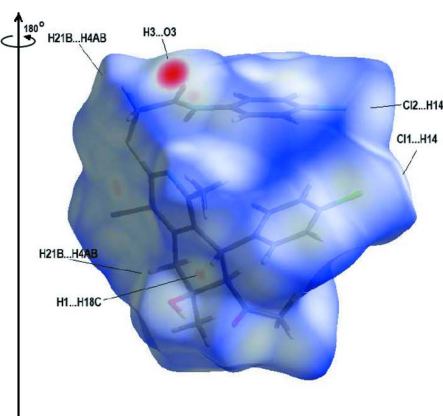

(b)
Figure 4

(a) Front and $(b)$ back sides of the three-dimensional Hirshfeld surface of the title compound plotted over $d_{\text {norm }}$ in the range -0.3918 to +1.6138 a.u.

\section{Database survey}

A survey of the Cambridge Structural Database (CSD, version 5.42, November 2020; Groom et al., 2016) reveals nine comparable tetrahydroisoquinoline derivatives, 7-acetyl-8-(4chlorophenyl)-3-(ethylsulfanyl)-6-hydroxy-1,6-dimethyl5,6,7,8-tetrahydroisoquinoline-4-carbonitrile (refcode NAQRIJ: Mague et al., 2017), 2-methyl-1,2,3,4-tetrahydroisoquinoline trihydrate (KUGLIK: Langenohl et al., 2020), 2'-benzoyl-1'-(4-methoxyphenyl)-1-methyl-1', $5^{\prime}, 6^{\prime}, 10^{\prime}$ b-tetrahydro-2' $H$-spiro[indole-3,3'-pyrrolo[2,1-a]isoquinolin]-2(1H)one (DUSVIZ: Selvaraj et al., 2020), 2-[(7-acetyl-4-cyano-6hydroxy-1,6-dimethyl-8-phenyl-5,6,7,8-tetrahydroisoquinolin3-yl)sulfanyl]- $N$-phenylacetamide (AKIVUO: Al-Taifi et al., 2021), 3-amino-1-oxo-2,6,8-triphenyl-1,2,7,8-tetrahydroiso-

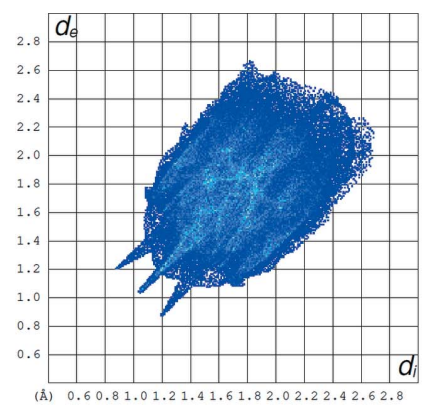

(a) All...All

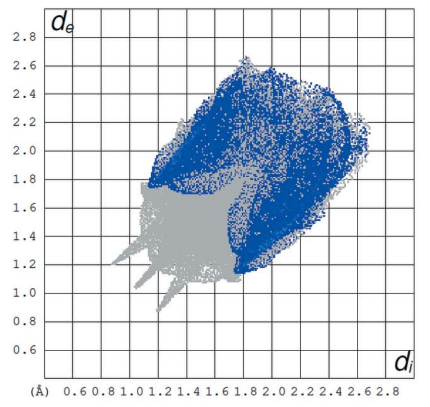

(c) $\mathrm{Cl} . . \mathrm{H} / \mathrm{H} \ldots \mathrm{Cl}$

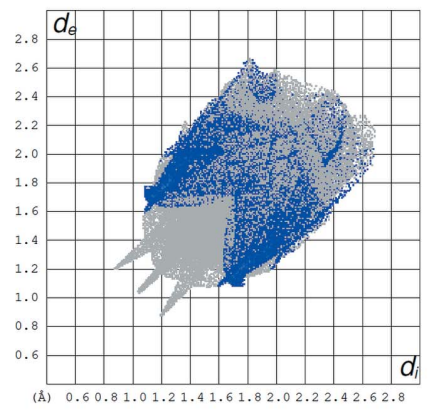

(e) $\mathrm{C} \ldots \mathrm{H} / \mathrm{H} \ldots \mathrm{C}$

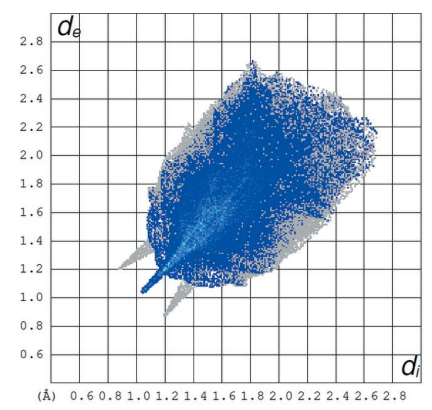

(b) $H$...H

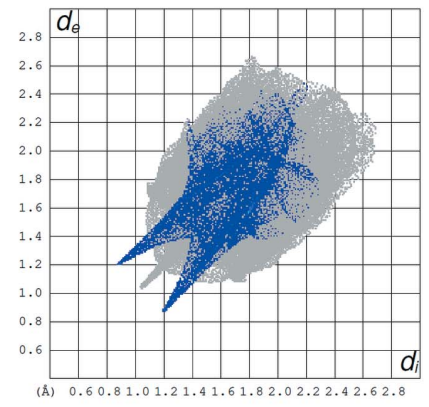

(d) $\mathrm{O} \ldots \mathrm{H} / \mathrm{H} \ldots \mathrm{O}$

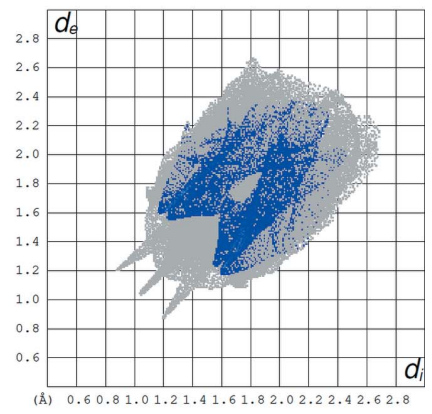

(f) $N \ldots H / H \ldots N$
Figure 5

A view of the two-dimensional fingerprint plots for the title compound, showing (a) all interactions, and delineated into $(b) \mathrm{H} \cdots \mathrm{H},(c) \mathrm{Cl} \cdots \mathrm{H} /$ $\mathrm{H} \cdots \mathrm{Cl},(d) \mathrm{O} \cdots \mathrm{H} / \mathrm{H} \cdots \mathrm{O},(e) \quad \mathrm{C} \cdots \mathrm{H} / \mathrm{H} \cdots \mathrm{C}$ and $(f) \quad \mathrm{N} \cdots \mathrm{H} / \mathrm{H} \cdots \mathrm{N}$ interactions. The $d_{\mathrm{i}}$ and $d_{\mathrm{e}}$ values are the closest internal and external distances (in $\AA$ ) from given points on the Hirshfeld surface. 
quinoline-4-carbonitrile (ULUTAZ: Naghiyev et al., 2021), 4-fluoro-3-(4-methoxyphenyl)-1-oxo-2-phenyl-1,2,3,4-tetrahydroisoquinoline-4-carboxylic acid (CARCOQ: Lehmann et al., 2017), 2-[3-methyl-4-phenyl-3,4-dihydroisoquinolin-2(1H)-yl]1,2-diphenylethan-1-ol (POPYEB: Ben Ali \& Retailleau, 2019), (1R,3S)-6,7-dimethoxy-3-(methoxydiphenylmethyl)-1phenyl-1,2,3,4-tetrahydroisoquinoline (ENOCIU: Naicker et al., 2011) and 1,2,3,4-tetrahydroisoquinoline-2-sulfonamide (NIWPAL: Bouasla et al., 2008).

In the crystal of NAQRIJ, dimers form through complementary sets of inversion-related $\mathrm{O}-\mathrm{H} \cdots \mathrm{O}$ and $\mathrm{C}-\mathrm{H} \cdots \mathrm{O}$ hydrogen bonds. These are connected into zigzag chains along the $c$-axis direction by pairwise $\mathrm{C}-\mathrm{H} \cdots \mathrm{N}$ interactions that also form inversion dimers. In the crystal of KUGLIK, the heterocyclic amines are alternately connected to the hydrogen-bonding system along the $c$ axis, which leads to the formation of syndiotactic polymer chains in this direction. The hydrogen-bonding network of the water molecules forms a water plane along the $b$ and $c$ axes with different ring systems (only counting the oxygen atoms) and graph-set motifs of the hydrogen-bonding network. In the crystal of DUSVIZ, molecules are linked via $\mathrm{C}-\mathrm{H} \cdots \mathrm{O}$ hydrogen bonds. For the major disorder component, these form $C(11)$ chains that propagate parallel to the $a$ axis. In the crystal of AKIVUO, a layer structure with the layers parallel to $(10 \overline{1})$ is generated by $\mathrm{O}-\mathrm{H} \cdots \mathrm{O}$ and $\mathrm{C}-\mathrm{H} \cdots \mathrm{O}$ hydrogen bonds. In the crystal of ULUTAZ, molecules are linked via $\mathrm{N}-\mathrm{H} \cdots \mathrm{O}$ and $\mathrm{C}-\mathrm{H} \cdots \mathrm{N}$ hydrogen bonds, forming a three-dimensional network. Furthermore, the crystal packing is dominated by $\mathrm{C}-\mathrm{H} \cdots \pi$ bonds with a strong interaction involving the phenyl $\mathrm{H}$ atoms. In the crystal of CARCOQ, molecules are linked by $\mathrm{O}-$ $\mathrm{H}$.. O hydrogen bonds, forming chains propagating along the $a$-axis direction. The chains are linked by $\mathrm{C}-\mathrm{H} \cdots \mathrm{F}$ hydrogen bonds, forming layers lying parallel to the $a b$ plane. In the crystal of POPYEB, molecules are packed in a herringbone manner parallel to (103) and $(10 \overline{3})$ via weak $\mathrm{C}-\mathrm{H} \cdots \mathrm{O}$ and $\mathrm{C}-\mathrm{H} \cdots \pi$ (ring) interactions. In the crystal structure of ENOCIU, various $\mathrm{C}-\mathrm{H} \cdots \pi$ and $\mathrm{C}-\mathrm{H} \cdots \mathrm{O}$ interactions link the molecules. In the crystal of NIWPAL, the molecules are linked by $\mathrm{N}-\mathrm{H}$. . O intermolecular hydrogen bonds involving the sulfonamide function to form an infinite two-dimensional network parallel to the (001) plane.
Table 4

Experimental details.

Crystal data

Chemical formula

$M_{\mathrm{r}}$

Crystal system, space group

Temperature (K)

$a, b, c(\AA)$

$\beta\left({ }^{\circ}\right)$

$V\left(\AA^{3}\right)$

Z

Radiation type

$\mu\left(\mathrm{mm}^{-1}\right)$

Crystal size (mm)

$\mathrm{C}_{28} \mathrm{H}_{25} \mathrm{Cl}_{2} \mathrm{~N}_{3} \mathrm{O}_{3} \mathrm{~S}$

554.47

Monoclinic, $P 2_{1} / c$

150

18.2076 (8), 14.2859 (6),

$10.2713(5)$

$98.245(1)$

$2644.1(2)$

4

Mo $K \alpha$

0.36

$0.29 \times 0.21 \times 0.17$

Data collection

Diffractometer

Absorption correction

$T_{\min }, T_{\max }$

No. of measured, independent and observed $[I>2 \sigma(I)]$ reflections

$R_{\text {int }}$

$(\sin \theta / \lambda)_{\max }\left(\AA^{-1}\right)$

Bruker SMART APEX CCD

Multi-scan (SADABS; Krause et al., 2015)

$0.85,0.94$

$50860,7143,5685$

0.037

0.689

Refinement

$R\left[F^{2}>2 \sigma\left(F^{2}\right)\right], w R\left(F^{2}\right), S$

No. of reflections

No. of parameters

$\mathrm{H}$-atom treatment

$\Delta \rho_{\max }, \Delta \rho_{\min }\left(\mathrm{e} \AA^{-3}\right)$

$0.041,0.115,1.07$

7143

337

$\mathrm{H}$-atom parameters constrained $0.55,-0.22$

Computer programs: APEX3 and SAINT (Bruker, 2016), SHELXT (Sheldrick, 2015a), SHELXL2018/1 (Sheldrick, 2015b), DIAMOND (Brandenburg \& Putz, 2012) and SHELXTL (Sheldrick, 2008).

\section{Synthesis and crystallization}

The title compound was obtained by refluxing of 7-acetyl-8-(4chlorophenyl)-4-cyano-1,6-dimethyl-6-hydroxy-5,6,7,8-tetrahydroisoquinoline-3(2H)-thione, $(0.77 \mathrm{~g}, 2 \mathrm{mmol})$ with $\mathrm{N}$-(4chlorophenyl)-2-chloroacetamide $(0.40 \mathrm{~g}, 2 \mathrm{mmol})$ and $(0.98 \mathrm{~g}, 12 \mathrm{mmol})$ of anhydrous sodium acetate in pure ethanol (30 ml) for $1 \mathrm{~h}$ as shown in Fig. 6. The product that formed during cooling was collected and recrystallized from ethanol to give good quality crystals suitable for X-ray diffraction. Yield: 1.00 g, 91\%; m.p. 491-493 K.

IR: $3522 \mathrm{~cm}^{-1}(\mathrm{O}-\mathrm{H}), 3277 \mathrm{~cm}^{-1}(\mathrm{~N}-\mathrm{H}), 2991,2920 \mathrm{~cm}^{-1}$ (C-H, aliphatic), $2217 \mathrm{~cm}^{-1}(\mathrm{C} \equiv \mathrm{N}), 1694(\mathrm{C}=\mathrm{O}$, acetyl),<smiles>CC(=O)C1C(c2ccc(Cl)cc2)c2c(C)[nH]c(=S)c(C#N)c2C[C@]1(C)O</smiles>
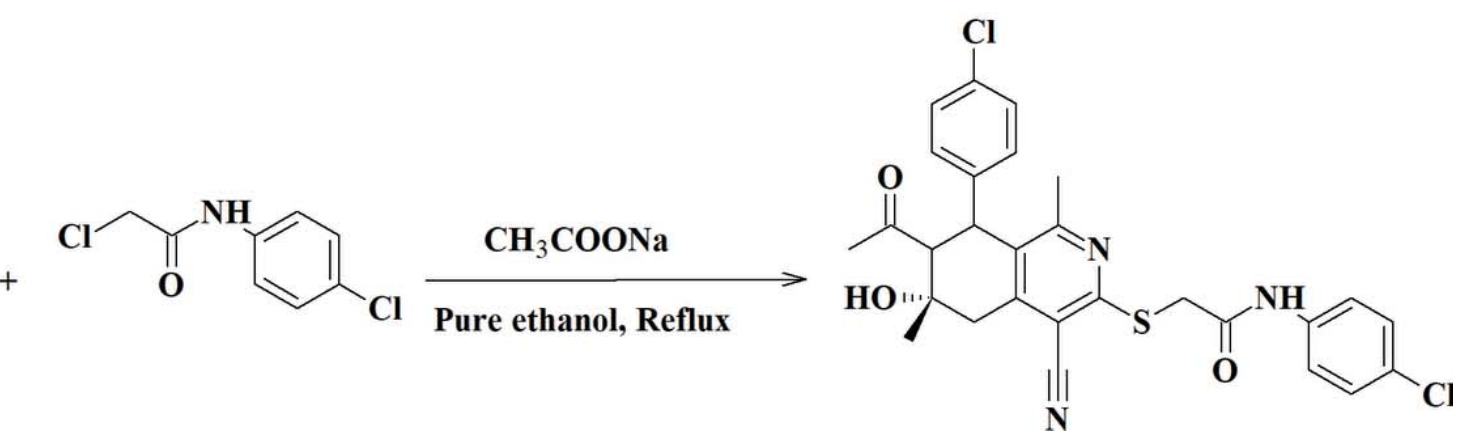

Figure 6

Synthesis scheme for 2-\{[7-acetyl-8-(4-chlorophenyl)-4-cyano-6-hydroxy-1,6-dimethyl-5,6,7,8-tetrahydroisoquinolin-3-yl] sulfanyl\}- $N$-(4-chlorophenyl)acetamide. 
$1666 \mathrm{~cm}^{-1}\left(\mathrm{C}=\mathrm{O}\right.$, amide). ${ }^{1} \mathrm{H}$ NMR (400 MHz, DMSO- $\left.d_{6}\right): \delta$ $10.95(s, 1 \mathrm{H}, \mathrm{NH}) ; 8.17-8.24(m, 2 \mathrm{H}, \mathrm{Ar}-\mathrm{H}) ; 7.79-7.81(d, 2 \mathrm{H}$, Ar-H); 7.26-7.32 (m, 2H, Ar-H); 7.03-7.05 (d, 2H, Ar-H); 4.88 $(s, 1 \mathrm{H}, \mathrm{OH}) ; 4.53-4.55(d, 1 \mathrm{H}, \mathrm{CH}$ at $\mathrm{C}-8) ; 4.19-4.20(d d, 2 \mathrm{H}$, $\left.\mathrm{SCH}_{2}\right)$; 3.24-3.29 (d, 1H, CH at C-5); 2.87-2.90 (m, 2H: $\mathrm{CH}$ at $\mathrm{C}-5$ and $\mathrm{CH}$ at $\mathrm{C}-7) ; 2.13\left(s, 3 \mathrm{H}, \mathrm{COCH}_{3}\right) ; 1.86\left(s, 3 \mathrm{H}, \mathrm{CH}_{3}\right.$ attached to pyridine ring); $1.27\left(s, 3 \mathrm{H}, \mathrm{CH}_{3}\right)$. Analysis calculated for $\mathrm{C}_{28} \mathrm{H}_{25} \mathrm{Cl}_{2} \mathrm{~N}_{3} \mathrm{O}_{3} \mathrm{~S}$ (554.47): $\mathrm{C} 60.65 \%, \mathrm{H} 4.54 \%, \mathrm{~N}$ $7.58 \%$, S 5.78\%. Found: C $60.34 \%, \mathrm{H} 4.57 \%$, N $7.68 \%, \mathrm{~S}$ $5.97 \%$.

\section{Refinement}

Crystal data, data collection and structure refinement details are summarized in Table 4. All C-bound $\mathrm{H}$ atoms were placed in geometrically idealized positions $(\mathrm{C}-\mathrm{H}=0.95-1.00 \AA)$ while those attached to $\mathrm{O}$ and to $\mathrm{N}$ were placed in locations derived from a difference map, refined for a few cycles to ensure that reasonable displacement parameters could be achieved, and then their coordinates were adjusted to give $\mathrm{O}-\mathrm{H}=0.87$ and $\mathrm{N}-\mathrm{H}=0.91 \AA$. All $\mathrm{H}$ atoms were included as riding contributions with isotropic displacement parameters 1.2-1.5 times those of the parent atoms.

\section{Acknowledgements}

Author contributions are as follows. Conceptualization, SKM and MA; methodology, ISM and JTM; investigation, ISM and JTM; writing (original draft), JTM, MA and SKM; writing (review and editing), AM and SKM; visualisation, SKM and AM; funding acquisition, SAHA; resources EAB, ISM and SAHA; supervision, AM, SKM and JTM.

\section{Funding information}

JTM thanks Tulane University for support of the Tulane Crystallography Laboratory.

\section{References}

Al-Taifi, E. A., Maraei, I. S., Bakhite, E. A., Demirtas, G., Mague, J. T., Mohamed, S. K. \& Ramli, Y. (2021). Acta Cryst. E77, 121-125. Ben Ali, K. \& Retailleau, P. (2019). Acta Cryst. E75, 1399-1402.
Bouasla, R., Berredjem, M., Aouf, N.-E. \& Barbey, C. (2008). Acta Cryst. E64, o432.

Brandenburg, K. \& Putz, H. (2012). DIAMOND, Crystal Impact GbR, Bonn, Germany.

Bruker (2016). APEX3 and SAINT. Bruker AXS Inc., Madison, Wisconsin, USA.

Chrzanowska, M., Schönenberger, B., Brossi, A. \& FlippenAnderson, J. L. (1987). Helv. Chim. Acta, 70, 1721-1731.

Cremer, D. \& Pople, J. A. (1975). J. Am. Chem. Soc. 97, 13541358.

Czarnocki, Z., Suh, D., MacLean, D. B., Hultin, P. G. \& Szarek, W. A. (1992). Can. J. Chem. 70, 1555-1561.

Gill, R., Brazell, C., Woodruff, G. N. \& Kemp, J. A. (1991). Br. J. Pharmacol. 103, 2030-2036.

Groom, C. R., Bruno, I. J., Lightfoot, M. P. \& Ward, S. C. (2016). Acta Cryst. B72, 171-179.

Houston, J. \& Rodger, I. (1974). Clin. Exp. Pharmacol. Physiol. 1, 401-413.

Krause, L., Herbst-Irmer, R., Sheldrick, G. M. \& Stalke, D. (2015). J. Appl. Cryst. 48, 3-10.

Langenohl, F., Otte, F. \& Strohmann, C. (2020). Acta Cryst. E76, 298302.

Lehmann, A., Lechner, L., Radacki, K., Braunschweig, H. \& Holzgrabe, U. (2017). Acta Cryst. E73, 867-870.

Mague, J. T., Mohamed, S. K., Akkurt, M., Bakhite, E. A. \& Albayati, M. R. (2017). IUCrData, 2, x170390.

McKinnon, J. J., Jayatilaka, D. \& Spackman, M. A. (2007). Chem. Commun. pp. 3814-3816.

Naghiyev, F. N., Grishina, M. M., Khrustalev, V. N., Khalilov, A. N., Akkurt, M., Akobirshoeva, A. A. \& Mamedov, I. G. (2021). Acta Cryst. E77, 195-199.

Naicker, T., Govender, T., Kruger, H. G. \& Maguire, G. E. M. (2011). Acta Cryst. C67, o100-o103.

Ohkubo, M., Kuno, A., Katsuta, K., Ueda, Y., Shirakawa, K., Nakanishi, H., Nakanishi, I., Kinoshita, T. \& Takasugi, H. (1996). Chem. Pharm. Bull. 44, 95-102.

Selvaraj, J. P., Mary, S., Dhruba, J. B., Huidrom, B. S., Panneerselvam, Y. \& Piskala Subburaman, K. (2020). Acta Cryst. E76, 1548-1550.

Sheldrick, G. M. (2008). Acta Cryst. A64, 112-122.

Sheldrick, G. M. (2015a). Acta Cryst. A71, 3-8.

Sheldrick, G. M. (2015b). Acta Cryst. C71, 3-8.

Spackman, M. A. \& Jayatilaka, D. (2009). CrystEngComm, 11, 1932.

Thompson, W. J., Anderson, P. S., Britcher, S. F., Lyle, T. A., Thies, J. E., Magill, C. A., Varga, S. L., Schwering, J. E., Lyle, P. A., Christy, M. E., et al. (1990). J. Med. Chem. 33, 789-808.

Turner, M. J., McKinnon, J. J., Wolff, S. K., Grimwood, D. J., Spackman, M. A., Jayatilaka, D. \& Spackman, M. A. (2017). Crystal Explorer. University of Western Australia. 


\section{supporting information}

Acta Cryst. (2021). E77, 527-531 [https://doi.org/10.1107/S2056989021003674]

Crystal structure and Hirshfeld surface analysis of 2-\{[7-acetyl-8-(4-chlorophenyl)-4-cyano-6-hydroxy-1,6-dimethyl-5,6,7,8-tetrahydroisoquinolin-3yl]sulfanyl\}- $N$-(4-chlorophenyl)acetamide

Mehmet Akkurt, Islam S. Marae, Joel T. Mague, Shaaban K. Mohamed, Etify A. Bakhite and Safiyyah A. H. Al-Waleedy

Computing details

Data collection: APEX3 (Bruker, 2016); cell refinement: SAINT (Bruker, 2016); data reduction: SAINT (Bruker, 2016); program(s) used to solve structure: SHELXT (Sheldrick, 2015a); program(s) used to refine structure: SHELXL2018/1 (Sheldrick, 2015b); molecular graphics: DIAMOND (Brandenburg \& Putz, 2012); software used to prepare material for publication: SHELXTL (Sheldrick, 2008).

2-\{[7-Acetyl-8-(4-chlorophenyl)-4-cyano-6-hydroxy-1,6-dimethyl-5,6,7,8-tetrahydroisoquinolin-3-yl]sulfanyl\}$N$-(4-chlorophenyl)acetamide

Crystal data

$\mathrm{C}_{28} \mathrm{H}_{25} \mathrm{Cl}_{2} \mathrm{~N}_{3} \mathrm{O}_{3} \mathrm{~S}$

$M_{r}=554.47$

Monoclinic, $P 2_{1} / c$

$a=18.2076(8) \AA$

$b=14.2859(6) \AA$

$c=10.2713(5) \AA$

$\beta=98.245(1)^{\circ}$

$V=2644.1(2) \AA^{3}$

$Z=4$

\section{Data collection}

\section{Bruker SMART APEX CCD} diffractometer

Radiation source: fine-focus sealed tube Graphite monochromator

Detector resolution: 8.3333 pixels $\mathrm{mm}^{-1}$ $\varphi$ and $\omega$ scans

Absorption correction: multi-scan (SADABS; Krause et al., 2015)

$T_{\min }=0.85, T_{\max }=0.94$

\section{Refinement}

Refinement on $F^{2}$ Least-squares matrix: full $R\left[F^{2}>2 \sigma\left(F^{2}\right)\right]=0.041$ $w R\left(F^{2}\right)=0.115$
$F(000)=1152$

$D_{\mathrm{x}}=1.393 \mathrm{Mg} \mathrm{m}^{-3}$

Mo $K \alpha$ radiation, $\lambda=0.71073 \AA$

Cell parameters from 9990 reflections

$\theta=2.5-29.3^{\circ}$

$\mu=0.36 \mathrm{~mm}^{-1}$

$T=150 \mathrm{~K}$

Column, colourless

$0.29 \times 0.21 \times 0.17 \mathrm{~mm}$

50860 measured reflections

7143 independent reflections

5685 reflections with $I>2 \sigma(I)$

$R_{\text {int }}=0.037$

$\theta_{\text {max }}=29.3^{\circ}, \theta_{\min }=1.8^{\circ}$

$h=-24 \rightarrow 25$

$k=-19 \rightarrow 19$

$l=-14 \rightarrow 14$

$S=1.07$

7143 reflections

337 parameters

0 restraints 
Primary atom site location: dual

Secondary atom site location: difference Fourier map

Hydrogen site location: mixed

$\mathrm{H}$-atom parameters constrained

$$
\begin{aligned}
& w=1 /\left[\sigma^{2}\left(F_{\mathrm{o}}^{2}\right)+(0.0726 P)^{2}+0.1632 P\right] \\
& \text { where } P=\left(F_{\mathrm{o}}^{2}+2 F_{\mathrm{c}}{ }^{2}\right) / 3 \\
& (\Delta / \sigma)_{\max }=0.001 \\
& \Delta \rho_{\max }=0.55 \mathrm{e} \AA^{-3} \\
& \Delta \rho_{\min }=-0.22 \mathrm{e} \AA^{-3}
\end{aligned}
$$

\section{Special details}

Experimental. The diffraction data were obtained from 3 sets of 400 frames, each of width $0.5^{\circ}$ in $\omega$, colllected at $\varphi=$ $0.00,90.00$ and $180.00^{\circ}$ and 2 sets of 800 frames, each of width $0.45^{\circ}$ in $\varphi$, collected at $\omega=-30.00$ and $210.00^{\circ}$. The scan time was $20 \mathrm{sec} /$ frame.

Geometry. All esds (except the esd in the dihedral angle between two 1.s. planes) are estimated using the full covariance matrix. The cell esds are taken into account individually in the estimation of esds in distances, angles and torsion angles; correlations between esds in cell parameters are only used when they are defined by crystal symmetry. An approximate (isotropic) treatment of cell esds is used for estimating esds involving l.s. planes.

Refinement. Refinement of $\mathrm{F}^{2}$ against ALL reflections. The weighted R-factor wR and goodness of fit $\mathrm{S}$ are based on $\mathrm{F}^{2}$, conventional R-factors $\mathrm{R}$ are based on $\mathrm{F}$, with $\mathrm{F}$ set to zero for negative $\mathrm{F}^{2}$. The threshold expression of $\mathrm{F}^{2}>2$ sigma( $\left.\mathrm{F}^{2}\right)$ is used only for calculating R-factors (gt) etc. and is not relevant to the choice of reflections for refinement. R-factors based on $\mathrm{F}^{2}$ are statistically about twice as large as those based on $\mathrm{F}$, and R- factors based on ALL data will be even larger. $\mathrm{H}$-atoms attached to carbon were placed in calculated positions $(\mathrm{C}-\mathrm{H}=0.95-1.00 \AA)$ while those attached to

\begin{tabular}{|c|c|c|c|c|}
\hline & $x$ & $y$ & $z$ & $U_{\text {iso }} * / U_{\text {eq }}$ \\
\hline $\mathrm{Cl1}$ & $-0.02990(2)$ & $0.36422(4)$ & 0.33858 & $0.04981(14)$ \\
\hline $\mathrm{Cl} 2$ & $0.06724(2)$ & $0.10721(4)$ & $0.37194(6)$ & $0.06092(16)$ \\
\hline $\mathrm{S} 1$ & $0.51601(2)$ & $0.33832(2)$ & $0.47637(3)$ & $0.02002(9)$ \\
\hline O1 & $0.18797(6)$ & $0.75204(7)$ & $0.15363(11)$ & $0.0362(2)$ \\
\hline $\mathrm{O} 2$ & $0.34473(5)$ & $0.71519(6)$ & $0.18526(9)$ & $0.0251(2)$ \\
\hline $\mathrm{H} 2 \mathrm{~A}$ & 0.306643 & 0.752176 & 0.183434 & $0.038 *$ \\
\hline $\mathrm{O} 3$ & $0.38487(5)$ & $0.25324(7)$ & $0.72696(9)$ & $0.0266(2)$ \\
\hline N3 & $0.38125(6)$ & $0.22031(7)$ & $0.50946(10)$ & $0.0202(2)$ \\
\hline H3 & 0.405602 & 0.226236 & 0.438511 & $0.024 *$ \\
\hline N1 & $0.39445(6)$ & $0.43662(7)$ & $0.50524(10)$ & $0.0195(2)$ \\
\hline N2 & $0.54848(7)$ & $0.44181(10)$ & $0.16630(12)$ & 0.0346 \\
\hline $\mathrm{C} 1$ & $0.25066(6)$ & $0.57818(8)$ & $0.28505(12)$ & $0.0182(2)$ \\
\hline H1 & 0.250642 & 0.638103 & 0.335370 & $0.022 *$ \\
\hline $\mathrm{C} 2$ & $0.24356(7)$ & $0.60275(8)$ & $0.13694(12)$ & $0.0192(2)$ \\
\hline $\mathrm{H} 2$ & 0.229573 & 0.544713 & 0.084806 & $0.023 *$ \\
\hline $\mathrm{C} 3$ & $0.31715(7)$ & $0.64019(9)$ & $0.09934(12)$ & $0.0193(2)$ \\
\hline $\mathrm{C} 4$ & $0.37436(7)$ & $0.56220(9)$ & $0.12178(12)$ & $0.0197(2)$ \\
\hline $\mathrm{H} 4 \mathrm{~A}$ & 0.362155 & 0.513961 & 0.052868 & $0.024 *$ \\
\hline H4AB & 0.423739 & 0.588126 & 0.112304 & $0.024 *$ \\
\hline $\mathrm{C} 5$ & $0.37853(6)$ & $0.51651(8)$ & $0.25450(11)$ & $0.0169(2)$ \\
\hline C6 & $0.44083(6)$ & $0.46236(8)$ & $0.30248(12)$ & $0.0175(2)$ \\
\hline $\mathrm{C} 7$ & $0.44414(6)$ & $0.41916(8)$ & $0.42543(12)$ & $0.0178(2)$ \\
\hline $\mathrm{C} 8$ & $0.33611(6)$ & $0.49192(8)$ & $0.46270(12)$ & $0.0189(2)$ \\
\hline C9 & $0.32273(6)$ & $0.52698(8)$ & $0.33326(11)$ & $0.0175(2)$ \\
\hline
\end{tabular}
nitrogen and to oxygen were placed in locations derived from a difference map and their coordinates adjusted to give $\mathrm{N}$ $-\mathrm{H}=0.91$ and $\mathrm{O}-\mathrm{H}=0.87 \% \mathrm{~A}$. All were included as riding contributions with isotropic displacement parameters 1.2 1.5 times those of the attached atoms.

Fractional atomic coordinates and isotropic or equivalent isotropic displacement parameters $\left(\AA^{2}\right)$ 


$\begin{array}{lllll}\text { C10 } & 0.18187(7) & 0.52296(9) & 0.30652(12) & 0.0210(2) \\ \text { C11 } & 0.17522(8) & 0.42867(10) & 0.27233(14) & 0.0270(3) \\ \text { H11 } & 0.215512 & 0.397364 & 0.241791 & 0.032^{*} \\ \text { C12 } & 0.11017(8) & 0.37991(11) & 0.28247(15) & 0.0335(3) \\ \text { H12 } & 0.105907 & 0.315681 & 0.258635 & 0.040^{*} \\ \text { C13 } & 0.05205(8) & 0.42535(12) & 0.32726(14) & 0.0331(3) \\ \text { C14 } & 0.05739(7) & 0.51863(12) & 0.36358(14) & 0.0329(3) \\ \text { H14 } & 0.017144 & 0.549228 & 0.395275 & 0.040^{*} \\ \text { C15 } & 0.12252(7) & 0.56685(10) & 0.35299(14) & 0.0273(3) \\ \text { H15 } & 0.126618 & 0.630890 & 0.377902 & 0.033^{*} \\ \text { C16 } & 0.18163(7) & 0.67507(10) & 0.10366(13) & 0.0249(3) \\ \text { C17 } & 0.11592(9) & 0.64770(13) & 0.00686(17) & 0.0427(4) \\ \text { H17A } & 0.131638 & 0.636429 & -0.079127 & 0.064^{*} \\ \text { H17B } & 0.079144 & 0.698219 & -0.000773 & 0.064^{*} \\ \text { H17C } & 0.093878 & 0.590491 & 0.036992 & 0.064^{*} \\ \text { C18 } & 0.30814(8) & 0.67229(10) & -0.04373(13) & 0.0281(3) \\ \text { H18A } & 0.288390 & 0.620672 & -0.101182 & 0.042^{*} \\ \text { H18B } & 0.356492 & 0.691427 & -0.066072 & 0.042^{*} \\ \text { H18C } & 0.273755 & 0.725379 & -0.055836 & 0.042^{*} \\ \text { C19 } & 0.50033(7) & 0.45056(9) & 0.22598(12) & 0.0216(3) \\ \text { C20 } & 0.28761(7) & 0.51467(10) & 0.56440(12) & 0.0259(3) \\ \text { H20A } & 0.267277 & 0.577862 & 0.548872 & 0.039^{*} \\ \text { H20B } & 0.316951 & 0.511668 & 0.652083 & 0.039^{*} \\ \text { H20C } & 0.246849 & 0.469388 & 0.558811 & 0.039^{*} \\ \text { C21 } & 0.48904(7) & 0.30133(9) & 0.63011(12) & 0.0211(2) \\ \text { H21A } & 0.526562 & 0.256096 & 0.671259 & 0.025^{*} \\ \text { H21B } & 0.491187 & 0.356564 & 0.688747 & 0.025^{*} \\ \text { C22 } & 0.41320(7) & 0.25678(8) & 0.62599(12) & 0.0200(2) \\ \text { C23 } & 0.30606(7) & 0.19224(9) & 0.48223(12) & 0.0208(2) \\ \text { C24 } & 0.28725(8) & 0.12164(10) & 0.38993(13) & 0.0261(3) \\ \text { H24 } & 0.324845 & 0.091634 & 0.349871 & 0.031^{*} \\ \text { C25 } & 0.21356(8) & 0.09505(10) & 0.35639(15) & 0.0323(3) \\ \text { H25 } & 0.200533 & 0.046510 & 0.294129 & 0.039^{*} \\ \text { C26 } & 0.15947(8) & 0.13995(11) & 0.41455(16) & 0.0338(3) \\ \text { C27 } & 0.17712(8) & 0.21117(11) & 0.50392(15) & 0.0321(3) \\ \text { H27 } & 0.139056 & 0.242487 & 0.541132 & 0.039^{*} \\ \text { C28 } & 0.25087(7) & 0.23702(10) & 0.53944(13) & 0.0263(3) \\ \text { H28 } & 0.263523 & 0.285112 & 0.602558 & 0.032^{*} \\ & & & & \end{array}$

Atomic displacement parameters $\left(\AA^{2}\right)$

\begin{tabular}{lllllll}
\hline & $U^{11}$ & $U^{22}$ & $U^{33}$ & $U^{12}$ & $U^{13}$ & $U^{23}$ \\
\hline C11 & $0.0360(2)$ & $0.0749(3)$ & $0.0390(2)$ & $-0.0295(2)$ & $0.00696(17)$ & $0.0015(2)$ \\
C12 & $0.0240(2)$ & $0.0690(3)$ & $0.0864(4)$ & $-0.01335(19)$ & $-0.0033(2)$ & $-0.0080(3)$ \\
S1 & $0.01734(15)$ & $0.01972(16)$ & $0.02272(16)$ & $0.00206(10)$ & $0.00195(11)$ & $0.00332(11)$ \\
O1 & $0.0341(6)$ & $0.0269(5)$ & $0.0470(6)$ & $0.0108(4)$ & $0.0042(5)$ & $0.0020(5)$ \\
O2 & $0.0255(5)$ & $0.0200(4)$ & $0.0296(5)$ & $-0.0013(3)$ & $0.0039(4)$ & $-0.0026(4)$ \\
O3 & $0.0287(5)$ & $0.0354(5)$ & $0.0161(4)$ & $0.0012(4)$ & $0.0043(4)$ & $0.0027(4)$
\end{tabular}




\begin{tabular}{|c|c|c|c|c|c|c|}
\hline N3 & $0.0210(5)$ & $0.0238(5)$ & $0.0162(5)$ & -0.0028 & $0.0042(4)$ & -0.0008 \\
\hline N1 & $0.0211(5)$ & $0.0195(5)$ & $0.0177(5)$ & $0.0002(4)$ & $0.0022(4)$ & $0.0015(4)$ \\
\hline $\mathrm{N} 2$ & $0.0323(7)$ & $0.0426(8)$ & $0.0314(7)$ & $0.0089(5)$ & $0.0125(5)$ & $0.0027(5)$ \\
\hline $\mathrm{C} 1$ & $0.0173(5)$ & $0.0184(6)$ & $0.0191(6)$ & $0.0012(4)$ & $0.0029(4)$ & $0.0008(4)$ \\
\hline $\mathrm{C} 2$ & $0.0197(6)$ & $0.0187(6)$ & $0.0184(6)$ & $0.0017(4)$ & $0.0006(4)$ & $0.0021(4)$ \\
\hline $\mathrm{C} 3$ & $0.0207(6)$ & $0.0193(6)$ & $0.0179(6)$ & $0.0017(4)$ & $0.0026(4)$ & $0.0019(4)$ \\
\hline $\mathrm{C} 4$ & $0.0209(6)$ & $0.0221(6)$ & $0.0164(5)$ & $0.0033(4)$ & $0.0043(4)$ & $0.0022(4)$ \\
\hline $\mathrm{C} 5$ & $0.0193(5)$ & $0.0158(5)$ & $0.0154(5)$ & $0.0000(4)$ & $0.0016(4)$ & -0.0003 \\
\hline C6 & $0.0184(5)$ & $0.0163(5)$ & $0.0182(5)$ & -0.0002 & $0.0040(4)$ & -0.0008 \\
\hline $\mathrm{C} 7$ & $0.0171(5)$ & $0.0165(5)$ & $0.0193(6)$ & 0.0004 (4) & 0.0005 (4) & $0.0010(4)$ \\
\hline $\mathrm{C} 8$ & $0.0199(5)$ & $0.0193(6)$ & $0.0178(5)$ & -0.0005 & $0.0031(4)$ & $0.0012(4)$ \\
\hline C9 & $0.0180(5)$ & $0.0168(5)$ & $0.0173(5)$ & $-0.0001(4)$ & 0.0015 (4) & $0.0002(4)$ \\
\hline $\mathrm{C} 10$ & $0.0187(6)$ & $0.0252(6)$ & $0.0190(6)$ & $0.0004(5)$ & $0.0022(4)$ & $0.0031(5)$ \\
\hline $\mathrm{C} 11$ & $0.0275(7)$ & $0.0259(7)$ & $0.0281(7)$ & $-0.0024(5)$ & $0.0057(5)$ & -0.0005 \\
\hline $\mathrm{C} 12$ & $0.0367(8)$ & $0.0324(8)$ & $0.0309(7)$ & $-0.0116(6)$ & $0.0034(6)$ & $0.0013(6)$ \\
\hline $\mathrm{C} 13$ & $0.0264(7)$ & $0.0466(9)$ & $0.0256(7)$ & $-0.0129(6)$ & $0.0018(5)$ & $0.0062(6)$ \\
\hline $\mathrm{C} 14$ & $0.0204(6)$ & $0.0488(9)$ & $0.0305(7)$ & $0.0008(6)$ & $0.0068(5)$ & $0.0044(6)$ \\
\hline $\mathrm{C} 15$ & $0.0228(6)$ & $0.0304(7)$ & $0.0292(7)$ & $0.0026(5)$ & $0.0058(5)$ & $0.0015(5)$ \\
\hline $\mathrm{C} 16$ & $0.0217(6)$ & $0.0304(7)$ & $0.0229(6)$ & $0.0051(5)$ & $0.0041(5)$ & $0.0082(5)$ \\
\hline C17 & $0.0278(8)$ & $0.0559(11)$ & $0.0405(9)$ & $0.0102(7)$ & $-0.0091(7)$ & $-0.0009(7)$ \\
\hline $\mathrm{C} 18$ & $0.0299(7)$ & $0.0321(7)$ & $0.0227(6)$ & $0.0062(5)$ & $0.0058(5)$ & $0.0106(5)$ \\
\hline C19 & $0.0241(6)$ & $0.0207(6)$ & $0.0199(6)$ & $0.0038(5)$ & $0.0026(5)$ & $0.0014(5)$ \\
\hline $\mathrm{C} 20$ & $0.0270(6)$ & $0.0322(7)$ & $0.0195(6)$ & $0.0072(5)$ & $0.0068(5)$ & $0.0040(5)$ \\
\hline $\mathrm{C} 21$ & $0.0219(6)$ & $0.0207(6)$ & $0.0195(6)$ & $0.0004(4)$ & $-0.0012(5)$ & $0.0025(5)$ \\
\hline $\mathrm{C} 22$ & $0.0234(6)$ & $0.0178(6)$ & $0.0181(6)$ & $0.0031(4)$ & $0.0010(4)$ & $0.0029(4)$ \\
\hline $\mathrm{C} 23$ & $0.0222(6)$ & $0.0218(6)$ & $0.0182(6)$ & $-0.0023(5)$ & $0.0023(4)$ & $0.0043(5)$ \\
\hline $\mathrm{C} 24$ & $0.0278(7)$ & $0.0265(7)$ & $0.0238(6)$ & $-0.0036(5)$ & $0.0025(5)$ & $-0.0012(5)$ \\
\hline $\mathrm{C} 25$ & $0.0324(7)$ & $0.0308(7)$ & $0.0318(7)$ & $-0.0082(6)$ & $-0.0014(6)$ & $-0.0025(6)$ \\
\hline $\mathrm{C} 26$ & $0.0231(7)$ & $0.0382(8)$ & $0.0384(8)$ & $-0.0073(6)$ & $-0.0016(6)$ & $0.0047(6)$ \\
\hline $\mathrm{C} 27$ & $0.0244(7)$ & $0.0371(8)$ & $0.0356(8)$ & $0.0010(6)$ & $0.0067(6)$ & $0.0032(6)$ \\
\hline $\mathrm{C} 28$ & $0.0261(7)$ & $0.0267(7)$ & $0.0263(6)$ & $0.0001(5)$ & $0.0045(5)$ & -0.0001 \\
\hline
\end{tabular}

Geometric parameters $\left(\AA,{ }^{\circ}\right)$

\begin{tabular}{llll}
\hline $\mathrm{C} 11-\mathrm{C} 13$ & $1.7471(14)$ & $\mathrm{C} 10-\mathrm{C} 11$ & $1.3929(18)$ \\
$\mathrm{C} 12-\mathrm{C} 26$ & $1.7376(14)$ & $\mathrm{C} 11-\mathrm{C} 12$ & $1.3907(19)$ \\
$\mathrm{S} 1-\mathrm{C} 7$ & $1.7674(12)$ & $\mathrm{C} 11-\mathrm{H} 11$ & 0.9500 \\
$\mathrm{~S} 1-\mathrm{C} 21$ & $1.7989(13)$ & $\mathrm{C} 12-\mathrm{C} 13$ & $1.376(2)$ \\
$\mathrm{O} 1-\mathrm{C} 16$ & $1.2120(18)$ & $\mathrm{C} 12-\mathrm{H} 12$ & 0.9500 \\
$\mathrm{O} 2-\mathrm{C} 3$ & $1.4325(15)$ & $\mathrm{C} 13-\mathrm{C} 14$ & $1.383(2)$ \\
$\mathrm{O} 2-\mathrm{H} 2 \mathrm{~A}$ & 0.8699 & $\mathrm{C} 14-\mathrm{C} 15$ & $1.3891(19)$ \\
$\mathrm{O} 3-\mathrm{C} 22$ & $1.2238(15)$ & $\mathrm{C} 14-\mathrm{H} 14$ & 0.9500 \\
$\mathrm{~N} 3-\mathrm{C} 22$ & $1.3576(15)$ & $\mathrm{C} 15-\mathrm{H} 15$ & 0.9500 \\
$\mathrm{~N} 3-\mathrm{C} 23$ & $1.4154(15)$ & $\mathrm{C} 16-\mathrm{C} 17$ & $1.494(2)$ \\
$\mathrm{N} 3-\mathrm{H} 3$ & 0.9096 & $\mathrm{C} 17-\mathrm{H} 17 \mathrm{~A}$ & 0.9800 \\
$\mathrm{~N} 1-\mathrm{C} 7$ & $1.3288(16)$ & $\mathrm{C} 17-\mathrm{H} 17 \mathrm{~B}$ & 0.9800 \\
$\mathrm{~N} 1-\mathrm{C} 8$ & $1.3454(15)$ & $\mathrm{C} 17-\mathrm{H} 17 \mathrm{C}$ & 0.9800 \\
$\mathrm{~N} 2-\mathrm{C} 19$ & $1.1464(17)$ & $\mathrm{C} 18-\mathrm{H} 18 \mathrm{~A}$ & 0.9800
\end{tabular}




\begin{tabular}{|c|c|c|c|}
\hline $\mathrm{C} 1-\mathrm{C} 9$ & $1.5219(16)$ & $\mathrm{C} 18-\mathrm{H} 18 \mathrm{~B}$ & 0.9800 \\
\hline $\mathrm{C} 1-\mathrm{C} 10$ & $1.5228(16)$ & $\mathrm{C} 18-\mathrm{H} 18 \mathrm{C}$ & 0.9800 \\
\hline $\mathrm{C} 1-\mathrm{C} 2$ & $1.5485(17)$ & $\mathrm{C} 20-\mathrm{H} 20 \mathrm{~A}$ & 0.9800 \\
\hline $\mathrm{C} 1-\mathrm{H} 1$ & 1.0000 & $\mathrm{C} 20-\mathrm{H} 20 \mathrm{~B}$ & 0.9800 \\
\hline $\mathrm{C} 2-\mathrm{C} 16$ & $1.5313(17)$ & $\mathrm{C} 20-\mathrm{H} 20 \mathrm{C}$ & 0.9800 \\
\hline $\mathrm{C} 2-\mathrm{C} 3$ & $1.5424(17)$ & $\mathrm{C} 21-\mathrm{C} 22$ & $1.5155(17)$ \\
\hline $\mathrm{C} 2-\mathrm{H} 2$ & 1.0000 & $\mathrm{C} 21-\mathrm{H} 21 \mathrm{~A}$ & 0.9900 \\
\hline $\mathrm{C} 3-\mathrm{C} 4$ & $1.5201(16)$ & $\mathrm{C} 21-\mathrm{H} 21 \mathrm{~B}$ & 0.9900 \\
\hline $\mathrm{C} 3-\mathrm{C} 18$ & $1.5256(17)$ & $\mathrm{C} 23-\mathrm{C} 28$ & $1.3906(19)$ \\
\hline $\mathrm{C} 4-\mathrm{C} 5$ & $1.5034(16)$ & $\mathrm{C} 23-\mathrm{C} 24$ & $1.3929(18)$ \\
\hline $\mathrm{C} 4-\mathrm{H} 4 \mathrm{~A}$ & 0.9900 & $\mathrm{C} 24-\mathrm{C} 25$ & $1.3894(19)$ \\
\hline $\mathrm{C} 4-\mathrm{H} 4 \mathrm{AB}$ & 0.9900 & $\mathrm{C} 24-\mathrm{H} 24$ & 0.9500 \\
\hline $\mathrm{C} 5-\mathrm{C} 9$ & $1.3945(17)$ & $\mathrm{C} 25-\mathrm{C} 26$ & $1.381(2)$ \\
\hline $\mathrm{C} 5-\mathrm{C} 6$ & $1.4027(16)$ & $\mathrm{C} 25-\mathrm{H} 25$ & 0.9500 \\
\hline $\mathrm{C} 6-\mathrm{C} 7$ & $1.3991(16)$ & $\mathrm{C} 26-\mathrm{C} 27$ & $1.377(2)$ \\
\hline C6- $-\mathrm{C} 19$ & $1.4365(17)$ & $\mathrm{C} 27-\mathrm{C} 28$ & $1.3901(19)$ \\
\hline $\mathrm{C} 8-\mathrm{C} 9$ & $1.4089(16)$ & $\mathrm{C} 27-\mathrm{H} 27$ & 0.9500 \\
\hline $\mathrm{C} 8-\mathrm{C} 20$ & $1.4971(18)$ & $\mathrm{C} 28-\mathrm{H} 28$ & 0.9500 \\
\hline $\mathrm{C} 10-\mathrm{C} 15$ & $1.3917(18)$ & & \\
\hline $\mathrm{C} 7-\mathrm{S} 1-\mathrm{C} 21$ & $99.68(6)$ & $\mathrm{C} 14-\mathrm{C} 13-\mathrm{Cl1}$ & $119.42(12)$ \\
\hline $\mathrm{C} 3-\mathrm{O} 2-\mathrm{H} 2 \mathrm{~A}$ & 103.5 & $\mathrm{C} 13-\mathrm{C} 14-\mathrm{C} 15$ & $118.97(13)$ \\
\hline $\mathrm{C} 22-\mathrm{N} 3-\mathrm{C} 23$ & $124.26(11)$ & $\mathrm{C} 13-\mathrm{C} 14-\mathrm{H} 14$ & 120.5 \\
\hline $\mathrm{C} 22-\mathrm{N} 3-\mathrm{H} 3$ & 118.3 & $\mathrm{C} 15-\mathrm{C} 14-\mathrm{H} 14$ & 120.5 \\
\hline $\mathrm{C} 23-\mathrm{N} 3-\mathrm{H} 3$ & 115.9 & $\mathrm{C} 14-\mathrm{C} 15-\mathrm{C} 10$ & $121.13(13)$ \\
\hline $\mathrm{C} 7-\mathrm{N} 1-\mathrm{C} 8$ & $119.05(10)$ & $\mathrm{C} 14-\mathrm{C} 15-\mathrm{H} 15$ & 119.4 \\
\hline $\mathrm{C} 9-\mathrm{C} 1-\mathrm{C} 10$ & $113.12(10)$ & $\mathrm{C} 10-\mathrm{C} 15-\mathrm{H} 15$ & 119.4 \\
\hline $\mathrm{C} 9-\mathrm{C} 1-\mathrm{C} 2$ & $112.04(10)$ & $\mathrm{O} 1-\mathrm{C} 16-\mathrm{C} 17$ & $122.54(13)$ \\
\hline $\mathrm{C} 10-\mathrm{C} 1-\mathrm{C} 2$ & $107.77(9)$ & $\mathrm{O} 1-\mathrm{C} 16-\mathrm{C} 2$ & $119.68(12)$ \\
\hline $\mathrm{C} 9-\mathrm{C} 1-\mathrm{H} 1$ & 107.9 & $\mathrm{C} 17-\mathrm{C} 16-\mathrm{C} 2$ & $117.76(12)$ \\
\hline $\mathrm{C} 10-\mathrm{C} 1-\mathrm{H} 1$ & 107.9 & $\mathrm{C} 16-\mathrm{C} 17-\mathrm{H} 17 \mathrm{~A}$ & 109.5 \\
\hline $\mathrm{C} 2-\mathrm{C} 1-\mathrm{H} 1$ & 107.9 & $\mathrm{C} 16-\mathrm{C} 17-\mathrm{H} 17 \mathrm{~B}$ & 109.5 \\
\hline $\mathrm{C} 16-\mathrm{C} 2-\mathrm{C} 3$ & $110.39(10)$ & $\mathrm{H} 17 \mathrm{~A}-\mathrm{C} 17-\mathrm{H} 17 \mathrm{~B}$ & 109.5 \\
\hline $\mathrm{C} 16-\mathrm{C} 2-\mathrm{C} 1$ & $109.11(10)$ & $\mathrm{C} 16-\mathrm{C} 17-\mathrm{H} 17 \mathrm{C}$ & 109.5 \\
\hline $\mathrm{C} 3-\mathrm{C} 2-\mathrm{C} 1$ & $111.81(10)$ & $\mathrm{H} 17 \mathrm{~A}-\mathrm{C} 17-\mathrm{H} 17 \mathrm{C}$ & 109.5 \\
\hline $\mathrm{C} 16-\mathrm{C} 2-\mathrm{H} 2$ & 108.5 & $\mathrm{H} 17 \mathrm{~B}-\mathrm{C} 17-\mathrm{H} 17 \mathrm{C}$ & 109.5 \\
\hline $\mathrm{C} 3-\mathrm{C} 2-\mathrm{H} 2$ & 108.5 & $\mathrm{C} 3-\mathrm{C} 18-\mathrm{H} 18 \mathrm{~A}$ & 109.5 \\
\hline $\mathrm{C} 1-\mathrm{C} 2-\mathrm{H} 2$ & 108.5 & $\mathrm{C} 3-\mathrm{C} 18-\mathrm{H} 18 \mathrm{~B}$ & 109.5 \\
\hline $\mathrm{O} 2-\mathrm{C} 3-\mathrm{C} 4$ & $106.43(9)$ & $\mathrm{H} 18 \mathrm{~A}-\mathrm{C} 18-\mathrm{H} 18 \mathrm{~B}$ & 109.5 \\
\hline $\mathrm{O} 2-\mathrm{C} 3-\mathrm{C} 18$ & $110.39(10)$ & $\mathrm{C} 3-\mathrm{C} 18-\mathrm{H} 18 \mathrm{C}$ & 109.5 \\
\hline $\mathrm{C} 4-\mathrm{C} 3-\mathrm{C} 18$ & $110.06(10)$ & $\mathrm{H} 18 \mathrm{~A}-\mathrm{C} 18-\mathrm{H} 18 \mathrm{C}$ & 109.5 \\
\hline $\mathrm{O} 2-\mathrm{C} 3-\mathrm{C} 2$ & $110.21(10)$ & $\mathrm{H} 18 \mathrm{~B}-\mathrm{C} 18-\mathrm{H} 18 \mathrm{C}$ & 109.5 \\
\hline $\mathrm{C} 4-\mathrm{C} 3-\mathrm{C} 2$ & $108.00(10)$ & $\mathrm{N} 2-\mathrm{C} 19-\mathrm{C} 6$ & $179.01(15)$ \\
\hline $\mathrm{C} 18-\mathrm{C} 3-\mathrm{C} 2$ & $111.60(10)$ & $\mathrm{C} 8-\mathrm{C} 20-\mathrm{H} 20 \mathrm{~A}$ & 109.5 \\
\hline $\mathrm{C} 5-\mathrm{C} 4-\mathrm{C} 3$ & $113.58(10)$ & $\mathrm{C} 8-\mathrm{C} 20-\mathrm{H} 20 \mathrm{~B}$ & 109.5 \\
\hline $\mathrm{C} 5-\mathrm{C} 4-\mathrm{H} 4 \mathrm{~A}$ & 108.8 & $\mathrm{H} 20 \mathrm{~A}-\mathrm{C} 20-\mathrm{H} 20 \mathrm{~B}$ & 109.5 \\
\hline $\mathrm{C} 3-\mathrm{C} 4-\mathrm{H} 4 \mathrm{~A}$ & 108.8 & $\mathrm{C} 8-\mathrm{C} 20-\mathrm{H} 20 \mathrm{C}$ & 109.5 \\
\hline $\mathrm{C} 5-\mathrm{C} 4-\mathrm{H} 4 \mathrm{AB}$ & 108.8 & $\mathrm{H} 20 \mathrm{~A}-\mathrm{C} 20-\mathrm{H} 20 \mathrm{C}$ & 109.5 \\
\hline
\end{tabular}




\begin{tabular}{|c|c|c|c|}
\hline $\mathrm{C} 3-\mathrm{C} 4-\mathrm{H} 4 \mathrm{AB}$ & 108.8 & $\mathrm{H} 20 \mathrm{~B}-\mathrm{C} 20-\mathrm{H} 20 \mathrm{C}$ & 109.5 \\
\hline $\mathrm{H} 4 \mathrm{~A}-\mathrm{C} 4-\mathrm{H} 4 \mathrm{AB}$ & 107.7 & $\mathrm{C} 22-\mathrm{C} 21-\mathrm{S} 1$ & $117.46(8)$ \\
\hline $\mathrm{C} 9-\mathrm{C} 5-\mathrm{C} 6$ & $118.36(10)$ & $\mathrm{C} 22-\mathrm{C} 21-\mathrm{H} 21 \mathrm{~A}$ & 107.9 \\
\hline $\mathrm{C} 9-\mathrm{C} 5-\mathrm{C} 4$ & $122.22(10)$ & $\mathrm{S} 1-\mathrm{C} 21-\mathrm{H} 21 \mathrm{~A}$ & 107.9 \\
\hline $\mathrm{C} 6-\mathrm{C} 5-\mathrm{C} 4$ & $119.41(10)$ & $\mathrm{C} 22-\mathrm{C} 21-\mathrm{H} 21 \mathrm{~B}$ & 107.9 \\
\hline $\mathrm{C} 7-\mathrm{C} 6-\mathrm{C} 5$ & $119.23(11)$ & $\mathrm{S} 1-\mathrm{C} 21-\mathrm{H} 21 \mathrm{~B}$ & 107.9 \\
\hline $\mathrm{C} 7-\mathrm{C} 6-\mathrm{C} 19$ & $120.24(10)$ & $\mathrm{H} 21 \mathrm{~A}-\mathrm{C} 21-\mathrm{H} 21 \mathrm{~B}$ & 107.2 \\
\hline $\mathrm{C} 5-\mathrm{C} 6-\mathrm{C} 19$ & $120.52(11)$ & $\mathrm{O} 3-\mathrm{C} 22-\mathrm{N} 3$ & $123.49(12)$ \\
\hline $\mathrm{N} 1-\mathrm{C} 7-\mathrm{C} 6$ & $121.94(11)$ & $\mathrm{O} 3-\mathrm{C} 22-\mathrm{C} 21$ & $119.13(11)$ \\
\hline $\mathrm{N} 1-\mathrm{C} 7-\mathrm{S} 1$ & $118.73(9)$ & $\mathrm{N} 3-\mathrm{C} 22-\mathrm{C} 21$ & $117.35(11)$ \\
\hline $\mathrm{C} 6-\mathrm{C} 7-\mathrm{S} 1$ & $119.32(9)$ & $\mathrm{C} 28-\mathrm{C} 23-\mathrm{C} 24$ & $119.91(12)$ \\
\hline $\mathrm{N} 1-\mathrm{C} 8-\mathrm{C} 9$ & $122.47(11)$ & $\mathrm{C} 28-\mathrm{C} 23-\mathrm{N} 3$ & $121.55(11)$ \\
\hline $\mathrm{N} 1-\mathrm{C} 8-\mathrm{C} 20$ & $114.72(10)$ & $\mathrm{C} 24-\mathrm{C} 23-\mathrm{N} 3$ & $118.43(12)$ \\
\hline $\mathrm{C} 9-\mathrm{C} 8-\mathrm{C} 20$ & $122.81(11)$ & $\mathrm{C} 25-\mathrm{C} 24-\mathrm{C} 23$ & $120.08(13)$ \\
\hline $\mathrm{C} 5-\mathrm{C} 9-\mathrm{C} 8$ & $117.88(10)$ & $\mathrm{C} 25-\mathrm{C} 24-\mathrm{H} 24$ & 120.0 \\
\hline $\mathrm{C} 5-\mathrm{C} 9-\mathrm{C} 1$ & $121.92(10)$ & $\mathrm{C} 23-\mathrm{C} 24-\mathrm{H} 24$ & 120.0 \\
\hline $\mathrm{C} 8-\mathrm{C} 9-\mathrm{C} 1$ & $120.15(11)$ & $\mathrm{C} 26-\mathrm{C} 25-\mathrm{C} 24$ & $119.27(13)$ \\
\hline $\mathrm{C} 15-\mathrm{C} 10-\mathrm{C} 11$ & $118.59(12)$ & $\mathrm{C} 26-\mathrm{C} 25-\mathrm{H} 25$ & 120.4 \\
\hline $\mathrm{C} 15-\mathrm{C} 10-\mathrm{C} 1$ & $120.76(12)$ & $\mathrm{C} 24-\mathrm{C} 25-\mathrm{H} 25$ & 120.4 \\
\hline $\mathrm{C} 11-\mathrm{C} 10-\mathrm{C} 1$ & $120.53(11)$ & $\mathrm{C} 27-\mathrm{C} 26-\mathrm{C} 25$ & $121.25(13)$ \\
\hline $\mathrm{C} 12-\mathrm{C} 11-\mathrm{C} 10$ & $120.64(14)$ & $\mathrm{C} 27-\mathrm{C} 26-\mathrm{Cl} 2$ & $119.44(12)$ \\
\hline $\mathrm{C} 12-\mathrm{C} 11-\mathrm{H} 11$ & 119.7 & $\mathrm{C} 25-\mathrm{C} 26-\mathrm{Cl} 2$ & $119.30(12)$ \\
\hline $\mathrm{C} 10-\mathrm{C} 11-\mathrm{H} 11$ & 119.7 & $\mathrm{C} 26-\mathrm{C} 27-\mathrm{C} 28$ & $119.71(14)$ \\
\hline $\mathrm{C} 13-\mathrm{C} 12-\mathrm{C} 11$ & $119.54(14)$ & $\mathrm{C} 26-\mathrm{C} 27-\mathrm{H} 27$ & 120.1 \\
\hline $\mathrm{C} 13-\mathrm{C} 12-\mathrm{H} 12$ & 120.2 & $\mathrm{C} 28-\mathrm{C} 27-\mathrm{H} 27$ & 120.1 \\
\hline $\mathrm{C} 11-\mathrm{C} 12-\mathrm{H} 12$ & 120.2 & $\mathrm{C} 27-\mathrm{C} 28-\mathrm{C} 23$ & $119.75(13)$ \\
\hline $\mathrm{C} 12-\mathrm{C} 13-\mathrm{C} 14$ & $121.12(13)$ & $\mathrm{C} 27-\mathrm{C} 28-\mathrm{H} 28$ & 120.1 \\
\hline $\mathrm{C} 12-\mathrm{C} 13-\mathrm{Cl} 1$ & $119.45(12)$ & $\mathrm{C} 23-\mathrm{C} 28-\mathrm{H} 28$ & 120.1 \\
\hline $\mathrm{C} 9-\mathrm{C} 1-\mathrm{C} 2-\mathrm{C} 16$ & $-165.26(10)$ & $\mathrm{C} 2-\mathrm{C} 1-\mathrm{C} 9-\mathrm{C} 5$ & $8.11(15)$ \\
\hline $\mathrm{C} 10-\mathrm{C} 1-\mathrm{C} 2-\mathrm{C} 16$ & $69.67(12)$ & $\mathrm{C} 10-\mathrm{C} 1-\mathrm{C} 9-\mathrm{C} 8$ & $-52.56(15)$ \\
\hline $\mathrm{C} 9-\mathrm{C} 1-\mathrm{C} 2-\mathrm{C} 3$ & $-42.86(13)$ & $\mathrm{C} 2-\mathrm{C} 1-\mathrm{C} 9-\mathrm{C} 8$ & $-174.62(11)$ \\
\hline $\mathrm{C} 10-\mathrm{C} 1-\mathrm{C} 2-\mathrm{C} 3$ & $-167.93(10)$ & $\mathrm{C} 9-\mathrm{C} 1-\mathrm{C} 10-\mathrm{C} 15$ & $137.21(12)$ \\
\hline $\mathrm{C} 16-\mathrm{C} 2-\mathrm{C} 3-\mathrm{O} 2$ & $69.67(12)$ & $\mathrm{C} 2-\mathrm{C} 1-\mathrm{C} 10-\mathrm{C} 15$ & $-98.37(13)$ \\
\hline $\mathrm{C} 1-\mathrm{C} 2-\mathrm{C} 3-\mathrm{O} 2$ & $-51.99(13)$ & $\mathrm{C} 9-\mathrm{C} 1-\mathrm{C} 10-\mathrm{C} 11$ & $-46.77(16)$ \\
\hline $\mathrm{C} 16-\mathrm{C} 2-\mathrm{C} 3-\mathrm{C} 4$ & $-174.46(10)$ & $\mathrm{C} 2-\mathrm{C} 1-\mathrm{C} 10-\mathrm{C} 11$ & $77.64(14)$ \\
\hline $\mathrm{C} 1-\mathrm{C} 2-\mathrm{C} 3-\mathrm{C} 4$ & $63.88(12)$ & $\mathrm{C} 15-\mathrm{C} 10-\mathrm{C} 11-\mathrm{C} 12$ & $1.0(2)$ \\
\hline $\mathrm{C} 16-\mathrm{C} 2-\mathrm{C} 3-\mathrm{C} 18$ & $-53.36(14)$ & $\mathrm{C} 1-\mathrm{C} 10-\mathrm{C} 11-\mathrm{C} 12$ & $-175.08(12)$ \\
\hline $\mathrm{C} 1-\mathrm{C} 2-\mathrm{C} 3-\mathrm{C} 18$ & $-175.02(10)$ & $\mathrm{C} 10-\mathrm{C} 11-\mathrm{C} 12-\mathrm{C} 13$ & $-0.3(2)$ \\
\hline $\mathrm{O} 2-\mathrm{C} 3-\mathrm{C} 4-\mathrm{C} 5$ & $69.02(12)$ & $\mathrm{C} 11-\mathrm{C} 12-\mathrm{C} 13-\mathrm{C} 14$ & $-0.6(2)$ \\
\hline $\mathrm{C} 18-\mathrm{C} 3-\mathrm{C} 4-\mathrm{C} 5$ & $-171.35(11)$ & $\mathrm{C} 11-\mathrm{C} 12-\mathrm{C} 13-\mathrm{Cl1}$ & $179.46(11)$ \\
\hline $\mathrm{C} 2-\mathrm{C} 3-\mathrm{C} 4-\mathrm{C} 5$ & $-49.30(13)$ & $\mathrm{C} 12-\mathrm{C} 13-\mathrm{C} 14-\mathrm{C} 15$ & $0.7(2)$ \\
\hline $\mathrm{C} 3-\mathrm{C} 4-\mathrm{C} 5-\mathrm{C} 9$ & $16.26(16)$ & $\mathrm{C} 11-\mathrm{C} 13-\mathrm{C} 14-\mathrm{C} 15$ & $-179.38(11)$ \\
\hline $\mathrm{C} 3-\mathrm{C} 4-\mathrm{C} 5-\mathrm{C} 6$ & $-162.89(11)$ & $\mathrm{C} 13-\mathrm{C} 14-\mathrm{C} 15-\mathrm{C} 10$ & $0.1(2)$ \\
\hline $\mathrm{C} 9-\mathrm{C} 5-\mathrm{C} 6-\mathrm{C} 7$ & $1.48(17)$ & $\mathrm{C} 11-\mathrm{C} 10-\mathrm{C} 15-\mathrm{C} 14$ & $-0.9(2)$ \\
\hline $\mathrm{C} 4-\mathrm{C} 5-\mathrm{C} 6-\mathrm{C} 7$ & $-179.33(11)$ & $\mathrm{C} 1-\mathrm{C} 10-\mathrm{C} 15-\mathrm{C} 14$ & $175.16(12)$ \\
\hline $\mathrm{C} 9-\mathrm{C} 5-\mathrm{C} 6-\mathrm{C} 19$ & $-179.09(11)$ & $\mathrm{C} 3-\mathrm{C} 2-\mathrm{C} 16-\mathrm{O} 1$ & $-59.41(16)$ \\
\hline $\mathrm{C} 4-\mathrm{C} 5-\mathrm{C} 6-\mathrm{C} 19$ & $0.10(17)$ & $\mathrm{C} 1-\mathrm{C} 2-\mathrm{C} 16-\mathrm{O} 1$ & $63.84(15)$ \\
\hline
\end{tabular}




$\begin{array}{llll}\mathrm{C} 8-\mathrm{N} 1-\mathrm{C} 7-\mathrm{C} 6 & 5.13(17) & \mathrm{C} 3-\mathrm{C} 2-\mathrm{C} 16-\mathrm{C} 17 & 119.10(13) \\ \mathrm{C} 8-\mathrm{N} 1-\mathrm{C} 7-\mathrm{S} 1 & -174.45(9) & \mathrm{C} 1-\mathrm{C} 2-\mathrm{C} 16-\mathrm{C} 17 & -117.65(14) \\ \mathrm{C} 5-\mathrm{C} 6-\mathrm{C} 7-\mathrm{N} 1 & -8.16(18) & \mathrm{C} 7-\mathrm{S} 1-\mathrm{C} 21-\mathrm{C} 22 & 59.47(10) \\ \mathrm{C} 19-\mathrm{C} 6-\mathrm{C} 7-\mathrm{N} 1 & 172.41(11) & \mathrm{C} 23-\mathrm{N} 3-\mathrm{C} 22-\mathrm{O} 3 & 14.1(2) \\ \mathrm{C} 5-\mathrm{C} 6-\mathrm{C} 7-\mathrm{S} 1 & 171.42(9) & \mathrm{C} 23-\mathrm{N} 3-\mathrm{C} 22-\mathrm{C} 21 & -167.77(11) \\ \mathrm{C} 19-\mathrm{C} 6-\mathrm{C} 7-\mathrm{S} 1 & -8.01(16) & \mathrm{S} 1-\mathrm{C} 21-\mathrm{C} 22-\mathrm{O} 3 & -160.99(10) \\ \mathrm{C} 21-\mathrm{S} 1-\mathrm{C} 7-\mathrm{N} 1 & 3.28(11) & \mathrm{S} 1-\mathrm{C} 21-\mathrm{C} 22-\mathrm{N} 3 & 20.84(15) \\ \mathrm{C} 21-\mathrm{S} 1-\mathrm{C} 7-\mathrm{C} 6 & -176.31(10) & \mathrm{C} 22-\mathrm{N} 3-\mathrm{C} 23-\mathrm{C} 28 & 31.66(18) \\ \mathrm{C} 7-\mathrm{N} 1-\mathrm{C} 8-\mathrm{C} 9 & 4.53(18) & \mathrm{C} 22-\mathrm{N} 3-\mathrm{C} 23-\mathrm{C} 24 & -152.12(12) \\ \mathrm{C} 7-\mathrm{N} 1-\mathrm{C} 8-\mathrm{C} 20 & -174.30(11) & \mathrm{C} 28-\mathrm{C} 23-\mathrm{C} 24-\mathrm{C} 25 & -0.9(2) \\ \mathrm{C} 6-\mathrm{C} 5-\mathrm{C} 9-\mathrm{C} 8 & 7.41(16) & \mathrm{N} 3-\mathrm{C} 23-\mathrm{C} 24-\mathrm{C} 25 & -177.19(12) \\ \mathrm{C} 4-\mathrm{C} 5-\mathrm{C} 9-\mathrm{C} 8 & -171.75(11) & \mathrm{C} 23-\mathrm{C} 24-\mathrm{C} 25-\mathrm{C} 26 & 0.6(2) \\ \mathrm{C} 6-\mathrm{C} 5-\mathrm{C} 9-\mathrm{C} 1 & -175.26(11) & \mathrm{C} 24-\mathrm{C} 25-\mathrm{C} 26-\mathrm{C} 27 & 0.7(2) \\ \mathrm{C} 4-\mathrm{C} 5-\mathrm{C} 9-\mathrm{C} 1 & 5.58(17) & \mathrm{C} 24-\mathrm{C} 25-\mathrm{C} 26-\mathrm{C} 2 & 179.63(11) \\ \mathrm{N} 1-\mathrm{C} 8-\mathrm{C} 9-\mathrm{C} 5 & -10.85(17) & \mathrm{C} 25-\mathrm{C} 26-\mathrm{C} 27-\mathrm{C} 28 & -1.8(2) \\ \mathrm{C} 20-\mathrm{C} 8-\mathrm{C} 9-\mathrm{C} 5 & 167.89(11) & \mathrm{C} 2-\mathrm{C} 26-\mathrm{C} 27-\mathrm{C} 28 & 179.33(11) \\ \mathrm{N} 1-\mathrm{C} 8-\mathrm{C} 9-\mathrm{C} 1 & 171.78(11) & \mathrm{C} 26-\mathrm{C} 27-\mathrm{C} 28-\mathrm{C} 23 & 1.5(2) \\ \mathrm{C} 20-\mathrm{C} 8-\mathrm{C} 9-\mathrm{C} 1 & -9.49(18) & \mathrm{C} 24-\mathrm{C} 23-\mathrm{C} 28-\mathrm{C} 27 & -0.1(2) \\ \mathrm{C} 10-\mathrm{C} 1-\mathrm{C} 9-\mathrm{C} 5 & 130.18(12) & \mathrm{N} 3-\mathrm{C} 23-\mathrm{C} 28-\mathrm{C} 27 & 176.03(12) \\ & & & \end{array}$

Hydrogen-bond geometry $\left(A,{ }^{\circ}\right)$

\begin{tabular}{lllll}
\hline$D-\mathrm{H} \cdots A$ & $D-\mathrm{H}$ & $\mathrm{H} \cdots A$ & $D \cdots A$ & $D-\mathrm{H} \cdots A$ \\
\hline $\mathrm{O} 2-\mathrm{H} 2 A \cdots \mathrm{O} 1$ & 0.87 & 2.14 & $2.8746(14)$ & 142 \\
$\mathrm{~N} 3-\mathrm{H} 3 \cdots \mathrm{O} 3^{\mathrm{i}}$ & 0.91 & 2.17 & $2.9362(13)$ & 141 \\
\hline
\end{tabular}

Symmetry code: (i) $x,-y+1 / 2, z-1 / 2$. 